\title{
Zum Zusammenspiel von Mathematikleistung, Schüler/innen- motivation und Lernemotionen auf der Sekundarstufe 1 - ein Vergleich zwischen Schüler/innen mit und ohne Migrationshintergrund in der Schweiz
}

\section{Claudia C. Brandenberger, Gerda Hagenauer und Tina Hascher}

Basierend auf dem Befund, dass Jugendliche mit Migrationshintergrund in der Schweiz geringere Leistungen erbringen, untersucht die vorliegende Studie, ob sich analoge Unterschiede auch in der Motivation und den Lernemotionen der Schülerlinnen, welche mit der Leistung zusammenhängen, zeigen. Weiter wird auf Grundlage der Kontroll-Wert-Theorie die Beziehung zwischen diesen Merkmalen und der Mathematikleistung überprüft. Die Ergebnisse ( $N=415$ Realschüler/innen der 7. Schulstufe) bestätigen die geringere Mathematikleistung von Schülerlinnen mit Migrationshintergrund bei einer gleichzeitig höher ausgeprägten selbstbestimmten Motivation und einem höheren Angsterleben. Hohe Leistung ist mit dem Fähigkeitsselbstkonzept in Mathematik assoziiert, welches wiederum ein positives emotionales Erlebensmuster (hohe Freude, geringe Angst) und die selbstbestimmte Motivation erklärt.

\section{Einleitung}

Durch die Ergebnisse der ersten PISA-Erhebung trat die Kluft in den Leistungen zwischen Schüler/innen mit und ohne Migrationshintergrund deutlich zu Tage und löste insbesondere im deutschsprachigen Raum bildungspolitische Debatten aus (Stanat, Rauch \& Segeritz, 2010). Nach der zweiten PISA-Welle im Jahr 2003, in der Mathematik den Schwerpunkt bildete, stellte sich heraus, dass nicht nur in Deutschland und Österreich, sondern auch in der Schweiz der Migrationshintergrund mit deutlichen Leistungsrückständen in Mathematik assoziiert ist (Moser, 2005; Stanat \& Christensen, 2006). Für Schüler/innen mit Migrationshintergrund (im Folgenden: SmM) in der Schweiz ist die Wahrscheinlichkeit, zur Risikogruppe in Mathematik zu gehören, 3.7-mal höher als für Schüler/innen ohne Migrationshintergrund (im Folgenden: SoM). Dieser Unterschied ist über alle OECD-Länder hinweg der grösste (Breit, 2009). Da schulische Leistungen nicht nur hinsichtlich der Selektion am Ende der Primarstufe, sondern auch bezüglich der zertifizierenden, nachobligatorischen Ausbildung weitreichende 
Konsequenzen haben und somit für die berufliche Zukunft von zentraler Bedeutung sind, erweisen sich diese Ergebnisse als besonders problematisch (Meyer, 2003)

Neben der kognitiven Leistungsfähigkeit stellen nicht-kognitive Merkmale zentrale Faktoren für erfolgreiche Bildungsprozesse dar. Insbesondere eine auf Selbstbestimmung beruhende Motivation erweist sich als wichtige Bedingungsvariable des Lernens (Deci \& Ryan, 1993). Positive Emotionen fördern, ähnlich wie die Motivation, das Lern- und Leistungsverhalten und sind darüber hinaus ein wichtiger Bestandteil des schulischen Wohlbefindens (Hascher \& Hagenauer, 2011; Schiepe-Tiska \& Schmidtner, 2013).

Die Frage nach den Bedingungen schulischer Motivation ist in Mathematik von besonderem Interesse, da die selbstbestimmte Motivation in diesem Fach über die Schulzeit hinweg, insbesondere im Verlauf der Sekundarstufe I, deutlich sinkt (Rakoczy, Klieme \& Pauli, 2008; Rheinberg \& Wendland, 2002). In gegliederten Schulsystemen weisen Schüler/innen, die den Schultyp mit den niedrigsten Anforderungen besuchen (= Hauptschule in Deutschland und Österreich bzw. Realniveau in der Schweiz), im Mittel die geringste Motivation auf (Fend, 1997).

Die vorliegende Untersuchung verfolgt daher das Ziel, die selbstbestimmte Motivation von Realschüler/innen der 7. Klassenstufe durch ausgewählte kognitive, motivationale und emotionale Schüler/innenmerkmale zu erklären sowie mögliche Unterschiede hinsichtlich dieser Variablen zwischen SmM und SoM aufzuzeigen. Die bisherigen empirischen Befunde zum Thema Migrationshintergrund und Schule verdeutlichen, dass der Migrationshintergrund mit zentralen motivationalen und kognitiven Komponenten des schulischen Lernens in Verbindung steht (Billmann-Mahecha \& Tiedemann, 2006; Meyer, 2003; Stanat \& Christensen, 2006). Diese Befunde sind jedoch uneinheitlich und variieren in Abhängigkeit der Stichprobe (Faber, Tiedemann \& Billmann-Mahecha, 2011; Roebers, Mecheril \& Schneider, 1998), der Leistungsdomäne (Schöber, Retelsdorf, \& Köller, 2015) sowie den berücksichtigten emotional-motivationalen Faktoren (Stanat, Segeritz, \& Christensen, 2010). Dabei wurden Lernemotionen im Vergleich zu anderen Merkmalen, wie z.B. das schulische Selbstkonzept, bisher wenig beachtet. Auch lässt sich spezifisch für die Schweiz ein Mangel an Untersuchungen zu migrationsbedingten Unterschieden hinsichtlich dieser Aspekte konstatieren, obwohl auf dem Realniveau der Sekundarstufe 1 ein Migrationsanteil von fast einem Viertel zu verzeichnen ist (Allraum, 2015).

\section{Theoretische Ansätze zur Erklärung von selbstbestimmter Motivation}

Die Selbstbestimmungstheorie der Motivation (SDT) (Deci \& Ryan, 1993) differenziert zwischen verschiedenen Formen schulischer Lernmotivation, die sich im Ausmass der erlebten Selbst- bzw. Fremdbestimmung unterscheiden. 
Selbstbestimmte Motivation liegt dann vor, wenn Schüler/innen intrinsisch motiviert lernen (der Lerngegenstand bzw. die Lernhandlung an sich ist motivierend) oder wenn eine identifizierte / integrierte Motivationslage vorliegt (die Schüler/innen lernen aus persönlicher Relevanz, häufig im Hinblick auf längerfristige Handlungsziele wie z.B. die berufliche Zukunft) (Deci \& Ryan, 1993; Müller, Hanfstingl \& Andreitz, 2007). Neben diesen beiden Formen der selbstbestimmten Motivation werden des Weiteren die externale (Schüler/innen lernen, um Belohnungen zu erhalten oder negative Konsequenzen zu vermeiden) und die introjizierte Regulation (Schüler/innen lernen "weil es sich gehört» oder um ein schlechtes Gewissen zu vermeiden) unterschieden, die höhere Anteile an Fremdbestimmung aufweisen. Im schulischen Kontext zeigt sich insbesondere die selbstbestimmte Motivation (repräsentiert in den Formen der intrinsischen sowie identifizierten Motivation) als förderlich für erfolgreiche Lernprozesse. Gemäss SDT kann effektives Lernen nur durch «ein vom individuellen Selbst ausgehendes Engagement erreicht werden» (Deci \& Ryan, 1993, S. 233). Neben der zentralen Bedeutung selbstbestimmter Motivation hinsichtlich lebenslangen Lernens zeigen empirische Befunde etwa, dass selbstbestimmte Formen der Motivation positiv mit positiven Emotionen (z.B. Lernfreude), dem schulischen Selbstkonzept, Interesse, Engagement und guten Leistungen assoziiert sind (Deci \& Ryan, 1993; Fend, 1997; Hagenauer, 2011). Angesichts ihrer bedeutsamen Rolle für das Lernen steht die selbstbestimmte Motivation im Fokus des vorliegenden Beitrags.

Die Entstehung von selbstbestimmter Motivation wird im Rahmen der SDT durch die Erfüllung der drei psychologischen Grundbedürfnisse (Autonomie, Kompetenz und soziale Eingebundenheit) erklärt (Deci \& Ryan, 2002): Werden diese Bedürfnisse beim Lernen erfüllt, so erleben die Lernenden den Ort der Handlungskontrolle eher als intern (statt extern), sie können die (vorgegebenen) Lernziele wertschätzen und ggf. in ihre eigene Zielhierarchie integrieren.

Als weiteres Rahmenmodell zur Erklärung der selbstbestimmten Motivation kann die Kontroll-Wert-Theorie (KWT) (Pekrun et al., 2002; Pekrun, 2006) herangezogen werden, die in der Emotionsforschung sehr häufig zur Anwendung kommt. Sie identifiziert zentrale kognitive und emotionale Schüler/innenmerkmale und deren Zusammenspiel, wodurch eine theoretische Modellableitung zur Vorhersage der selbstbestimmten Motivation ermöglicht wird. Basierend auf einem appraisal-orientierten Ansatz der Emotionsentstehung (Shuman \& Scherer, 2014) postuliert die KWT, dass die subjektive Bewertung der Umwelt die Qualität der jeweiligen Emotion im Lehr-Lernkontext bestimmt. Als zentral werden die Kontroll- und Wertkognitionen erachtet. Erleben Schüler/innen die schulische Umwelt als kontrollierbar (= Kontrollkognition) und als intrinsisch oder extrinsisch «wertvoll» (z.B. die Lerninhalte sind relevant und/oder interessant; = Wertkognition), so entstehen typischerweise positive Emotionen. Auch Leistungssituationen können als ein Umweltmerkmal interpretiert werden, das von Schüler/innen bewertet wird. Gemäss 
des «skill-development»-Ansatzes (Hosenfeld, 2002) wird angenommen, dass gute Leistungen das Fähigkeitsselbstkonzept (als Indikator für eine Kontrollkognition) stärken. Ein hohes Fähigkeitsselbstkonzept fördert das Erleben positiver Lern- und Leistungsemotionen und vermindert negative Emotionen (Götz et al., 2004). Positive Emotionen wiederum gehen mit einer hohen selbstbestimmten Motivation einher (Pekrun \& Götz, 2006; Pekrun et al., 2002). Die Zusammenhänge zwischen den Merkmalen sind komplex, da sowohl direkte als auch indirekte Effekte angenommen werden. Des Weiteren ist von reziproken Beziehungen auszugehen (z.B. Götz et al., 2004; siehe auch Abbildung 1).

Die KWT geht davon aus, dass die Zusammenhänge zwischen diesen Merkmalen Allgemeingültigkeit besitzen, sprich geschlechts-, kultur- und herkunftsunabhängig sind (Pekrun, 2006). Es wird folglich angenommen, dass das Beziehungsgefüge zwischen Leistung, Fähigkeitsselbstkonzept, Emotion und Motivation für unterschiedliche Gruppen gleichermassen gilt; unabhängig von der mittleren Ausprägung der einzelnen Schüler/innenmerkmale. Auf Basis der KWT ist daher beispielsweise davon auszugehen, dass eine gute Leistung in Mathematik grundsätzlich bei Schüler/innen ein positives Fähigkeitsselbstkonzept begünstigt; auch dann, wenn die durchschnittliche Leistung von SmM geringer ist als von SoM.

\section{Empirische Befundlage: Schüler/innenmerkmale und Migrationshintergrund \\ Selbstbestimmte Lernmotivation}

Angesichts des reziproken Zusammenhangs zwischen der Leistung und der Motivation (Mega, Ronconi \& De Beni, 2014) wäre anzunehmen, dass sich bei SmM weniger positive motivationale Einstellungen ausbilden, da diese überzufällig häufig Defizite in Mathematik aufweisen. Entsprechende Befunde zeichnen jedoch ein in dieser Hinsicht erwartungswidriges Bild: Vertiefende Analysen der PISA 2003-Erhebung von Stanat und Christensen (2006) ergeben, dass SmM im Vergleich zu SoM in den meisten Ländern ein ähnliches, wenn nicht sogar höheres mathematikbezogenes Motivationsniveau aufweisen. Ebenso unterscheiden sich Schüler/innen der ersten Generation ${ }^{1}$ in der Schweiz von Schüler/innen der zweiten Generation und zwar im Sinne einer höheren intrinsischen und instrumentellen (identifizierten) Motivation zu Gunsten der ersten Generation (Stanat \& Christensen, 2006). Ähnliche Befunde für Deutschland zeigen sich in der MARKUS-Studie: SmM (8. Klassenstufe) erzielen zwar ungünstigere Leistungen; ihre intrinsische und instrumentelle (identifizierte) Lernmotivation fällt aber deutlich höher aus als bei SoM (Helmke, Hosenfeld, Schrader \& Wagner, 2002). Erklärungen dafür liefert der Immigrant Optimism, welcher postuliert, dass Migrant/innen in besonderem Masse dazu tendieren, schulisch erfolgreich sein zu wollen, da die Migration als Möglichkeit des sozialen Aufstiegs erachtet wird (Stanat, Segeritz \& Christensen, 2010). Dementsprechend sollten insbesondere Schüler/innen spät zugewanderter Familien (Schüler/innen der 
ersten Generation) eine hohe schulische Motivation und hohe Bildungsaspirationen aufweisen. Studien, die migrationsspezifische Muster hinsichtlich der extrinischen Motivation gemäss SDT (externale und introjizierte Motivation) untersuchen, liegen allerdings kaum vor. Eine Ausnahme bildet die Untersuchung von Buholzer, Ottinger und von Büren (2008), in der analog zu den Befunden zur selbstbestimmten Formen der Motivation aufgezeigt werden konnte, dass SmM, insbesondere Jungen, stärker extrinsisch motiviert lernen als SoM.

\section{Lernemotionen}

Auch schulbezogene Emotionen beeinflussen das Lern- und Leistungsverhalten (Schiepe-Tiska \& Schmidtner, 2013). Dabei haben sich im Hinblick auf die Schulleistung folgende Emotionen als besonders relevant herausgestellt: die Angst und die Lernfreude (Schrader \& Helmke, 2002). Entsprechend der Klassifikation von Pekrun et al. (2002) zählen sie zu den Lern- und Leistungsemotionen («academic emotions» oder «achievement emotions»), die in schulischen Kontexten hervorgerufen werden.

Zum Zusammenhang zwischen dem Migrationshintergrund und der allgemeinen Leistungs- bzw. Prüfungsangst kommen Studien zum Ergebnis, dass SmM häufiger (allgemeine) Leistungsangst erleben (Jerusalem, 1988; Roebers \& Schneider, 1999). Im Hinblick auf die Angst vor Mathematik konnten Stanat und Christensen (2006) anhand der PISA-2003-Daten zeigen, dass in allen Ländern, ausser in Belgien, ebenfalls deutliche Unterschiede zu Ungunsten der SmM bestehen. Als Erklärung für diese Befunde kann der bedeutsame Zusammenhang zwischen der Angst vor Mathematik und den Mathematikleistungen herangezogen werden: Schwächere Mathematikleistungen gehen in der Regel mit höherer Angst vor Mathematik einher (Bauer, Ramseier \& Blum, 2014). In Übereinstimmung mit den Befunden der mathematikbezogenen Motivation kommen Untersuchungen zur (Lern-)Freude in Mathematik jedoch auch zum Ergebnis, dass SmM mehr Freude berichten als SoM (Faber et al., 2011; Stanat \& Christensen, 2006).

\section{Fähigkeitsselbstkonzept}

In engem Zusammenhang mit den Mathematikleistungen, der Motivation und den Emotionen steht das mathematische Fähigkeitsselbstkonzept, definiert als die subjektive Einschätzung der eigenen Lern- und Leistungsfähigkeit in Mathematik (Bauer et al., 2014). Ausgehend von reziproken positiven Beziehungen zwischen schulischen Leistungen und Fähigkeitsselbstkonzepten liegt die Vermutung nahe, dass SmM ein schwächeres Fähigkeitsselbstkonzept in Mathematik entwickeln, da sie oftmals weniger erfolgreich sind als einheimische Schüler/innen (Stanat \& Christensen, 2006). Studien zum allgemeinen schulischen Selbstkonzept zeigen jedoch kein einheitliches Bild. Während etwa die frühe Studie von Schwarzer, Lange und Jerusalem (1981) die Annahme eines 
geringeren Selbstkonzepts bei SmM in Deutschland bestätigen, ergibt sich bei Billmann-Mahecha und Tiedemann (2006) ein gegenteiliger Befund: SmM in Deutschland weisen ein optimistischeres Fähigkeitsselbstkonzept auf, als es auf Grund ihrer Schulleistungen zu erwarten wäre. Obwohl neben dem allgemeinen schulischen Selbstkonzept auch domänenspezifische schulische Selbstkonzepte als zentrale Einflussfaktoren auf schulische Leistungen gelten (Schöber et al., 2015), existieren nur wenige Studien, die das mathematische Fähigkeitsselbstkonzept bei SmM auf der Sekundarstufe I unter Berücksichtigung des Migrationsstatus der Kinder untersuchen. Die Ergebnisse dieser Studien zeigen, dass SmM ein insgesamt höheres Fähigkeitsselbstkonzept aufweisen als SoM (Faber et al., 2011; Helmke et al., 2002; Shajek, Lüdtke \& Stanat, 2006). Dies gilt insbesondere für Schüler/innen der ersten Generation und wird damit begründet, dass diese Schüler/innen in Mathematik im Gegensatz zu Deutsch, wo ihre Lesekompetenzen stärker durch ihre Sprachdefizite beeinträchtigt sind, trotz geringeren Leistungen eher das Gefühl haben, gute Leistungen erbringen zu können (Lehmann, Gänsfuss \& Peek, 2011). Entsprechend belegen Studien von Roebers und Schneider (1999) und Shajek et al. (2006), dass innerhalb der Gruppe von Jugendlichen mit Migrationshintergrund diejenigen ein höheres Fähigkeitsselbstkonzept in Mathematik haben, die über geringere Kenntnisse der deutschen Sprache verfügen.

\section{Zielsetzung und Hypothesen}

Die Zusammenschau der empirischen Befunde hat gezeigt, dass bisher nur vereinzelte Befunde - vor allem für die Schweiz - zum Zusammenhang der angeführten Schüler/innenmerkmale unter Berücksichtigung des Faktors «Migrationshintergrund» existieren. Die vorliegende Studie verfolgt daher zwei Ziele: In einem ersten Schritt sollen zunächst Unterschiede zwischen SmM und SoM im Hinblick auf die Mathematikleistung, die Motivation, die erlebten Emotionen sowie das mathematische Fähigkeitsselbstkonzept analysiert werden. Des Weiteren interessieren Unterschiede zwischen der ersten und zweiten Generation innerhalb der Gruppe von SmM. Folgende Hypothesen lassen sich aufgrund der bisherigen Befundlage ableiten:

(1) SmM erzielen niedrigere Mathematiktestleistungen als SoM.

(2) SmM verfügen über (a) eine höhere selbstbestimmte Motivation (b) eine höhere Lernfreude, (c) eine höhere Angst und (d) ein höheres Fähigkeitsselbstkonzept in Mathematik als SoM.

(3) Innerhalb der Gruppe der Jugendlichen mit Migrationshintergrund unterscheiden sich Schüler/innen der ersten Generation von Schüler/innen der zweiten Generation hinsichtlich der genannten motivationalen und emotionalen Faktoren sowie der Testleistung. Die Hypothese wird aufgrund der bisher geringen empirischen Evidenz bewusst ungerichtet formuliert. 
In einem zweiten Schritt wird der Zusammenhang zwischen den Merkmalen auf Basis der KWT getestet (siehe Abbildung 1).

Basierend auf dem «skill development»-Ansatz wird erwartet, dass gute Leistungen das Fähigkeitsselbstkonzept stärken und damit positive Emotionen (Freude) fördern und negative Emotionen (Angst) mindern. Ein positives emotionales Erlebensmuster (hohe Freude, geringe Angst) wiederum begünstigt die selbstbestimmte Motivation. Es werden sowohl direkte als auch indirekte Effekte zwischen den Konstrukten angenommen.

Gemäss KWT wird des Weiteren erwartet, dass die angeführten Zusammenhänge zwischen den Merkmalen gleichermassen für SmM und für SoM gelten. Da migrationsspezifische Muster aber durch herkunftsbedingte Unterschiede geprägt sein können, wird der sozioökonomische Status kontrolliert.

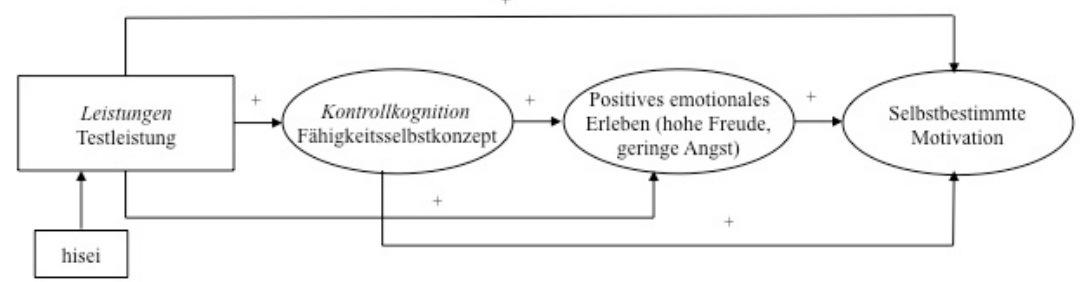

Abbildung 1: Modell zur Erklärung der selbstbestimmten Motivation

\section{Methode}

\section{St ichprobe}

Die zur Beantwortung der Forschungsfragen verwendeten Daten stammen aus dem vom Schweizerischen Nationalfonds geförderten Projekt «Maintaining and Fostering Students' Positive Learning Emotions and Learning Motivation in Math Instruction During Adolescence» (Projektnummer: 100019_156710). Im Herbst 2015 wurden 415 Realschüler/innen der 7. Klassenstufe aus insgesamt 23 Klassen (17 Schulen; Kanton Bern) mittels eines Fragebogens durch geschulte Testleiter/innen befragt. Wie bereits einführend erwähnt, wird diese Altersgruppe als besonders kritisch bezogen auf den Rückgang von positiven emotional-motivationalen Lernfaktoren erachtet. Insgesamt weisen $34.9 \%(\mathrm{n}=145)$ Schüler/innen einen Migrationshintergrund (erste Generation, $\mathrm{n}=35$ (8.4\%); zweite Generation, $\mathrm{n}=110(26.5 \%)$ ) auf. Das Geschlechterverhältnis ist mit $50.4 \%(\mathrm{n}=209)$ Mädchen und $49.6 \%(\mathrm{n}=206)$ Jungen ausgeglichen. Das Durchschnittsalter beträgt 12.75 Jahre $(S D=0.64)$. 


\section{Erhobene Variablen}

Die selbstbestimmte Motivation in Mathematik setzt sich aus den beiden Subskalen intrinsische und identifizierte Motivation zusammen (Müller et al., 2007). Die intrinsische Motivation wurde anhand von fünf Items erfasst (z.B. «Ich arbeite und lerne in Mathematik, weil ich gerne Aufgaben in Mathematik löse», $\alpha=.87$ ); die identifizierte Motivation anhand von vier Items (z.B. «Ich arbeite und lerne in Mathematik, weil ich damit mehr Möglichkeiten bei der späteren Berufswahl habe», $\alpha=.84,1$ = stimmt überhaupt nicht, 5 = stimmt völlig).

Die Emotionen im Mathematikunterricht wurden mit Hilfe einer gekürzten Version des «Achievement Emotions Questionnaire - Mathematics (AEQ-M) - German» (Pekrun, Götz \& Frenzel, 2005) erhoben. Die Emotionen Freude und Angst wurden auf einer fünfstufigen Skala $(1=$ stimmt gar nicht, $5=$ stimmt genau) zu je vier Items erfasst (z.B. "Der Mathematikunterricht macht mir Spass»; "Aus Angst vor Mathematik würde ich am liebsten nicht in die Schule gehen»). Die interne Konsistenz der Skalen erwies sich als zufriedenstellend ( $\alpha=.92$ für Freude und $\alpha=.86$ für Angst).

Das mathematische Fähigkeitsselbstkonzept (Skala aus PISA 2012) (Bauer, Ramseier \& Blum, 2014; Schwantner, Toferer \& Schreiner, 2013) wurde mit fünf Items (z.B. «Mathematik ist eines meiner besten Fächer») auf einem vierstufigem Antwortformat ( $1=$ trifft nicht zu, $4=$ trifft zu) gemessen $(\alpha=.86)$.

Zur Erfassung der Mathematikleistung wurden Testbögen aus HarmoS $2007^{2}$ übernommen, welche für Schüler/innen am Ende von Klasse 6 konzipiert und in dieser Untersuchung zu Beginn des 7. Schuljahres eingesetzt wurden (Wälti, 2014). Die Schüler/innen erhielten vier Testbögen zu den Themen funktionale Zusammenhänge (Testbogen 1), Zahl und Variablen (Testbogen 2 und 4) sowie Masse und Grössen (Testbogen 3), wobei sie mindestens drei, idealerweise alle vier bearbeiten sollten. Die Schüler/innen hatten die Wahl, in welcher Reihenfolge sie vorgehen wollten. Die Aufgaben wurden bei der Konzipierung so ausgewählt, dass jeweils mindestens eine Aufgabe aus jedem Feld der heuristischen Matrix des Lehrplans 21 vertreten ist. Die Testleistungen orientieren sich dabei an der bereits bestehenden Skala von HarmoS $2007(M=500, S D=100)$ für Schüler/innen am Ende der 6. Klassenstufe (Wälti, 2014).

Der Migrationshintergrund wurde über die Schülerangaben zum Geburtsland der Schüler/innen sowie ihrer Eltern bestimmt. In den Analysen werden analog zu den PISA-Studien folgende Kategorien unterschieden (Bauer et al., 2014): Die Kategorie 1 bilden SmM. Sie umfasst (a) Schüler/innen der «ersten Generation», d.h. im Ausland geborene Schüler/innen mit ebenfalls im Ausland geborenen Eltern sowie (b) Schüler/innen der "zweiten Generation", mit in der Schweiz geborenen Schüler/innen, deren beider Elternteile jedoch im Ausland geboren sind. Alle anderen Schüler/innen werden in PISA als SoM bezeichnet und bilden die Kategorie 2. Diese Kategorie umfasst somit die in der Schweiz geborenen Schüler/innen mit mindestens einem in der Schweiz geborenen Elternteil, sowie die im Ausland geborenen Schüler/innen mit mindestens einem in der Schweiz geborenen Elternteil. 
Weiter wurde der sozioökonomische Status der Herkunftsfamilie berücksichtigt. Dieser wurde über die Angaben der Berufe der Eltern erhoben. Die Berufsangaben der Eltern wurden anhand der International Standard Classification of Occupation (ISCO-88(COM)) klassifiziert und anschliessend mittels International Socio-Economic Index (ISEI) in eine metrische Skala transformiert (Ganzeboom, De Graaf \& Treiman, 1992). In die Analysen floss bei differierenden Indexausprägungen für Mutter und Vater der HISEI (Highest ISEI) ein, der den höchsten Wert der Familie berücksichtigt $($ Min $=16, \operatorname{Max}=88$, $M=42.94, S D=14.23$ ).

\section{Datenanalyse}

Die Datenanalyse erfolgte mit Hilfe der Software SPSS (Version 23.0) sowie Mplus (Version 7.3) (Muthén \& Muthén, 1998-2012). Für den Vergleich der Mittelwerte aller untersuchten Variablen zwischen SoM und SmM sowie zwischen SmM der ersten und zweiten Generation kamen $t$-Tests für unabhängige Stichproben zur Anwendung (Alpha-Level = $5 \%$ ). Als Effektstärke wurde Cohen's $d$ berechnet. Da die Stichprobe vor allem in der Gruppe der SmM der ersten Generation relativ klein ist, kommt der Interpretation der Effektstärke eine besondere Bedeutung zu.

Zur Modelltestung wurden Strukturgleichungsmodelle spezifiziert. Basierend auf der theoretischen Konzeptualisierung von selbstbestimmter Motivation durch die SDT bildeten die Indikatoren «intrinsische und identifizierte Motivation» das Konstrukt der selbstbestimmten Motivation. Das positive emotionale Erlebensmuster der Schüler/innen wurde durch die Skalen «Freude» und "Angst» (negativer Indikator) modelliert, um zwei zentrale Lernund Leistungsemotionen abzubilden. Zur Überprüfung der Modellgüte wurde auf folgende Fit-Indices Bezug genommen: Comparative Fit-Index (CFI) und Tucker-Lewis-Index (TLI), deren Werte über .95 liegen sollten, sowie Root-Mean-Square-Error-of-Approximation (RMSEA) und Standardized-Root-Mean-Square-Residual (SRMR) mit Kriteriumswerten unter .05 (Geiser, 2010). Die Daten unterliegen einer hierarchischen Struktur. Da für die vorliegende Untersuchung keine Variablen auf Klassenebene in die Analysen einbezogen werden, sondern lediglich Zusammenhänge zwischen Variablen auf Individualebene interessieren, wurde jedoch keine 2. Ebene spezifiziert. Allerdings wurden robuste Standardfehler (Cluster Robust Standard Errors; TYPE=COMPLEX) geschätzt. Missings (>1.2 \% für Motivation, Emotionen, Fähigkeitsselbstkonzept; $5.3 \%$ für HISEI; $7.5 \%$ für Mathematiktest wurden mit Hilfe der Full-Information Maximum Likelihood Schätzung (FIML) ersetzt. 


\section{Ergebnisse}

Deskriptive Statistiken und Gruppenunterschiede

Die deskriptiven Statistiken, differenziert nach SmM und SoM, sowie die Korrelationen zwischen den Variablen finden sich in Tabelle 1. Die Korrelationen bestätigen überwiegend die positiven Beziehungen zwischen den Konstrukten.

Bezogen auf die erwarteten Unterschiede zwischen SmM und SoM fallen hypothesenkonform die mittleren Mathematikleistungen $\left(\mathrm{H}_{1}\right)$ von $\mathrm{SmM}$ signifikant niedriger aus als die Leistungen von SoM. Werden die vier verschiedenen Themenbereiche des Leistungstests betrachtet, so zeigen sich hinsichtlich des Themenbereichs «Zahl und Variablen» (Testbogen 2+4) keine Unterschiede zwischen SmM und SoM. Bedeutsame Unterschiede ergeben sich hingegen bei «funktionale Zusammenhänge» (Testbogen $1, t(382)=2.64 p<.01$ ) und «Masse und Grössen» (Testbogen 3, $t(382)=4.34 p<.001)$ : In den Bereichen «funktionale Zusammenhänge» und «Masse und Grössen» erreichen SmM signifikant tiefere Leistungen als SoM. Diese Ergebnisse sind jedoch mit Vorsicht $\mathrm{zu}$ interpretieren, da Schüler/innen, welche eine Aufgabe nicht gelöst, und Schüler/innen, welche eine Aufgabe falsch gelöst, gleichermassen null Punkte erhalten hatten.

Im Einklang mit den Hypothesen sind ebenso die Mittelwertsunterschiede zwischen den beiden Gruppen hinsichtlich der selbstbestimmten Motivation $\left(\mathrm{H}_{2 \mathrm{a}}\right)$ und der Angst $\left(\mathrm{H}_{2 \mathrm{c}}\right)$. SmM geben demnach an, höher intrinsisch sowie identifiziert motiviert zu sein. Gleichzeitig ist ihre mathematikbezogene Angst höher. Wider Erwarten unterscheiden sich die SmM nicht signifikant von den SoM hinsichtlich der Freude $\left(\mathrm{H}_{2 \mathrm{~b}}\right)$ und dem Fähigkeitsselbstkonzept $\left(\mathrm{H}_{2 \mathrm{~d}}\right)$. Die diesbezüglichen Effektstärken sind ebenso unbedeutend.

Wird die Gruppe der SmM nach Generation differenziert betrachtet (siehe Tabelle 2), so fällt zunächst auf, dass sich die Mittelwerte der beiden Gruppen in allen untersuchten Variablen unterscheiden, wobei die Unterschiede jedoch eher gering sind, ausgenommen das Ausmass der selbstberichteten Angst: Schüler/innen der ersten Generation äussern eine höhere Angst. Dieser Unterschied ist mit einer Effektstärke von $d=-.37$ substanziell, lässt sich jedoch nicht statistisch auf einem 5\%-igen Signifikanzlevel absichern. Für alle weiteren Merkmale sind die Unterschiede deutlich geringer und ebenfalls nicht signifikant. Schüler/innen mit Migrationshintergrund der ersten Generation berichten ein höheres Fähigkeitsselbstkonzept und eine bessere Leistung; die intrinsische Motivation ist jedoch geringer. Diese Effekte liegen auf einem Niveau von .10 $<d<.15$. Es lässt sich folglich kein klares Muster in den zentralen emotional-motivationalen Merkmalen zwischen Schüler/innen der ersten und zweiten Generation identifizieren $\left(\mathrm{H}_{3}\right)$. Zu berücksichtigen ist bei diesen Ergebnissen die geringe Stichprobengrösse in der Gruppe der Schüler/innen der ersten Generation. 
Tabelle 1: Mittelwerte, Gruppenunterschiede ( $t$-Werte und Cohen's d) und Korrelationsmatrix der erhobenen Skalen

\begin{tabular}{|c|c|c|c|c|c|c|c|c|c|c|c|c|c|c|c|}
\hline \multirow[b]{2}{*}{ Variable } & \multicolumn{3}{|c|}{$\begin{array}{c}\text { Schüler/innen } \\
\text { mit Migra- } \\
\text { tionshintergrund }\end{array}$} & \multicolumn{3}{|c|}{$\begin{array}{c}\text { Schüler/innen } \\
\text { ohne Migra- } \\
\text { tionshintergrund }\end{array}$} & \multirow[b]{2}{*}{$\mathrm{t}$} & \multirow[b]{2}{*}{$\mathrm{d}$} & \multirow[b]{2}{*}{1} & \multirow[b]{2}{*}{2} & \multirow[b]{2}{*}{3} & \multirow[b]{2}{*}{4} & \multirow[b]{2}{*}{5} & \multirow[b]{2}{*}{6} & \multirow[b]{2}{*}{7} \\
\hline & $\mathrm{N}$ & M & SD & $\mathrm{N}$ & M & SD & & & & & & & & & \\
\hline $\begin{array}{l}\text { 1. Intrinsische } \\
\text { Motivation }\end{array}$ & 145 & 3.20 & 0.86 & 268 & 2.97 & 0.95 & $-2.56^{* *}$ & -0.25 & - & $.42^{* *}$ & $.81^{* *}$ & $-.17^{*}$ & $.66^{* *}$ & $.15^{* *}$ & -.08 \\
\hline $\begin{array}{l}\text { 2. Identifizierte } \\
\text { Motivation }\end{array}$ & 145 & 4.17 & 0.84 & 268 & 3.95 & 0.87 & $-2.46^{* *}$ & -0.26 & & - & $.39^{* *}$ & -.02 & $.31^{* *}$ & $.13^{*}$ & .00 \\
\hline 3. Freude & 144 & 3.12 & 1.08 & 268 & 3.03 & 1.08 & -0.79 & -0.08 & & & - & $-.28^{* *}$ & $.68^{* *}$ & $.21^{* *}$ & -.01 \\
\hline 4. Angst & 144 & 2.15 & 1.01 & 268 & 1.88 & 0.87 & $-2.72^{* *}$ & -0.29 & & & & - & $-.24^{* *}$ & $-.20^{* *}$ & .05 \\
\hline $\begin{array}{l}\text { 5. Fähigkeits- } \\
\text { selbstkonzept }\end{array}$ & 145 & 2.43 & 0.74 & 268 & 2.40 & 0.70 & -0.46 & -0.04 & & & & & - & $.21^{* *}$ & -.01 \\
\hline 6. Testleistung & 125 & 420 & 66.75 & 259 & 442 & 57.01 & $3.05^{* *}$ & 0.37 & & & & & & - & .08 \\
\hline 7. HISEI & 129 & 38.2 & 13.45 & 264 & 45.3 & 14.15 & $4.72^{* * *}$ & 0.51 & & & & & & & - \\
\hline
\end{tabular}

${ }^{*} \mathrm{p}<.05 ;{ }^{* *} \mathrm{p}<.01 ;{ }^{* * *} \mathrm{p}<.001$

Tabelle 2: Mittelwerte und Gruppenunterschiede von Schülerlinnen mit Migrationshintergrund, differenziert nach erste und zweite Generation

\begin{tabular}{|c|c|c|c|c|c|c|c|c|}
\hline \multirow[b]{2}{*}{ Variable } & \multicolumn{3}{|c|}{$\begin{array}{l}\text { Schüler/innen der } \\
\text { ersten Generation }\end{array}$} & \multicolumn{3}{|c|}{$\begin{array}{c}\text { Schüler/innen der zweiten } \\
\text { Generation }\end{array}$} & \multirow[b]{2}{*}{$\mathrm{t}$} & \multirow[b]{2}{*}{ d } \\
\hline & $\mathrm{N}$ & M & SD & $\mathrm{N}$ & M & SD & & \\
\hline 1. Intrinsische Motivation & 35 & 3.11 & 0.94 & 110 & 3.24 & 0.84 & -0.76 & 0.15 \\
\hline 2. dentifizierte Motivation & 35 & 4.14 & 0.79 & 110 & 4.18 & 0.86 & -0.23 & 0.05 \\
\hline 3. Freude & 35 & 3.09 & 1.11 & 109 & 3.13 & 1.07 & -0.17 & 0.04 \\
\hline 4. Angst & 35 & 2.43 & 1.06 & 109 & 2.06 & 0.98 & 1.90 & $-0.37^{+}$ \\
\hline 5. Fähigkeitsselbstkonzept & 35 & 2.50 & 0.73 & 110 & 2.41 & 0.74 & 0.58 & -0.12 \\
\hline 6. Testleistung & 31 & 426 & 78 & 94 & 419 & 63 & 0.52 & -0.11 \\
\hline 7. HISEI & 27 & 40.30 & 15.70 & 102 & 37.65 & 12.82 & 0.81 & -0.20 \\
\hline
\end{tabular}

$+\mathrm{p}<.10 ;{ }^{*} \mathrm{p}<.05 ;{ }^{* *} \mathrm{p}<.01 ;{ }^{* * *} \mathrm{p}<.001$

Vorhersage der selbstbestimmten Motivation

Die Berechnung des Strukturgleichungsmodells ergibt einen zufriedenstellenden Datenfit $\left(\chi^{2}(52)=76.478, \mathrm{p}<.001\right.$; CFI $=.977$, TLI $=.968$, RMSEA $=.050$, SRMR =.053). Das Modell ist in Abbildung 2 dargestellt, wobei die Pfadkoeffizienten (standardisierte Beta-Koeffizienten) immer zuerst für SmM und danach für SoM angegeben sind. 
Erwartungskonform stehen hohe Testleistungen in Mathematik mit dem Fähigkeitsselbstkonzept $\left(\mathrm{R}^{2}=.08\right)$ in positiver Beziehung $(\mathrm{H} 4)$. Das Fähigkeitsselbstkonzept erweist sich wiederum als prädiktiv für die erlebten Emotionen $\left(\mathrm{R}^{2}\right.$ $=.38$ für SmM; $\mathrm{R}^{2}=.43$ für SoM). Schüler/innen mit einem positiven Fähigkeitsselbstkonzept erleben folglich auch eine höhere Freude und weniger Angst. Der positive Zusammenhang zwischen den Emotionen und der selbstbestimmten Motivation $\left(\mathrm{R}^{2}=.74\right)$ impliziert: Je positiver das emotionale Erlebensmuster im Mathematikunterricht erlebt werden, desto höher fällt die selbstbestimmte Motivation aus. Diese Zusammenhänge gelten sowohl für SmM als auch für SoM (H5). Die selbstbestimmte Motivation wird bei SmM besonders stark durch ihr Fähigkeitsselbstkonzept, bei SoM durch die Emotionen erklärt. Entgegen der Erwartungen ergeben sich für beide Gruppen keine signifikant direkten Effekte zwischen der Testleistung und der selbstbestimmten Motivation sowie den Emotionen.

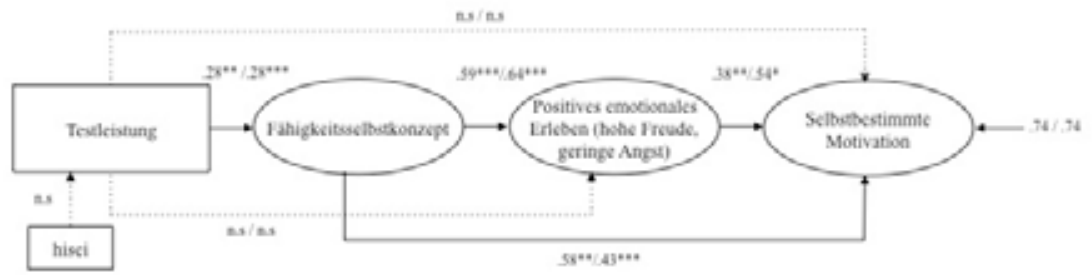

Abbildung 2: Beziehung zwischen der Schulleistung, dem Fähigkeitsselbstkonzept, dem positiven emotionalen Erleben (hohe Freude, geringe Angst) und der selbstbestimmten Motivation unter Kontrolle des sozioökonomischen Status (linker Pfadkoeffizient = SmM; rechter Pfadkoeffizient $=$ SoM)

In Tabelle 3 sind die direkten und indirekten Effekte im Überblick dargestellt. Es zeigt sich, dass der indirekte Effekt der Mathematikleistung auf die selbstbestimmte Motivation vor allem über das Fähigkeitsselbstkonzept vermittelt wird.

Tabelle 3: Standardisierte indirekte Effekte und Standardfehler im Gesamtmodell

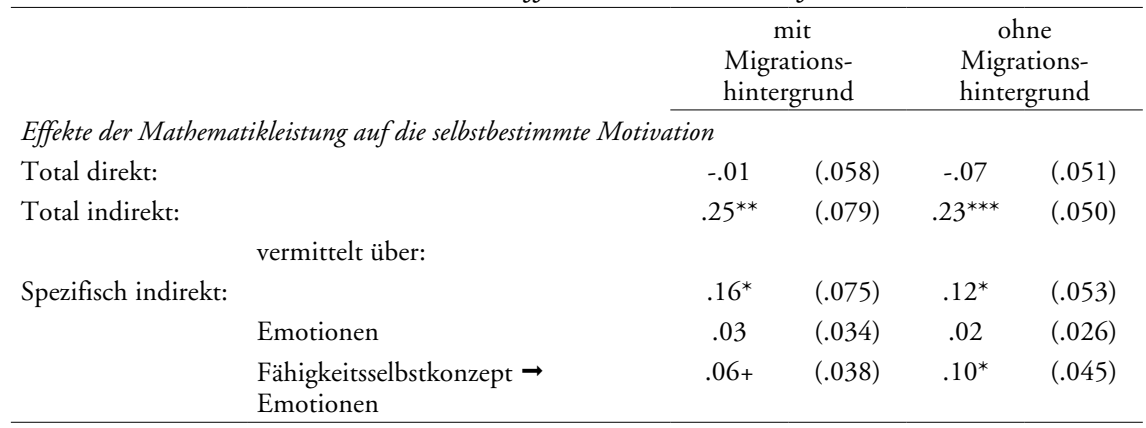




\begin{tabular}{|c|c|c|c|c|c|}
\hline \multirow{2}{*}{\multicolumn{2}{|c|}{ Effekte der Mathematikleistung auf die Emotione }} & \multicolumn{2}{|c|}{$\begin{array}{c}\text { mit } \\
\text { Migrations- } \\
\text { hintergrund }\end{array}$} & \multicolumn{2}{|c|}{$\begin{array}{l}\text { ohne } \\
\text { Migrations- } \\
\text { hintergrund }\end{array}$} \\
\hline & & & & & \\
\hline Total direkt: & & .07 & $(.074)$ & .03 & $(.047)$ \\
\hline Total indirekt: & Fähigkeitsselbstkonzept & $.16^{*}$ & $(.065)$ & $.18^{* * *}$ & $(.045)$ \\
\hline \multicolumn{6}{|c|}{ Effekte des Fähigkeitsselbstkonzepts auf die selbstbestimmte Motivation } \\
\hline Total direkt: & & $.58^{* * *}$ & $(.136)$ & $.43^{* *}$ & $(.162)$ \\
\hline Total indirekt: & Emotionen & .23 & $(.139)$ & $.35^{*}$ & $(.145)$ \\
\hline
\end{tabular}

Anmerkungen. Werte in Klammern: Standardfehler $+\mathrm{p}<.10 ;{ }^{*} \mathrm{p}<.05 ;{ }^{* *} \mathrm{p}<.01 ;{ }^{* * *} \mathrm{p}<.001$

\section{Diskussion}

Der vorliegende Beitrag verfolgte das Ziel, den Forschungsstand über SmM in der Schweiz im Hinblick auf zentrale Komponenten schulischen Lernens in Mathematik (Leistungen, Fähigkeitsselbstkonzept, erlebte Emotionen und selbstbestimmte Motivation) zu erweitern und mit SoM zu vergleichen. Ebenso wurde in Anlehnung an die KWT (Pekrun, 2006) die Beziehung zwischen der Schulleistung und den emotional-motivationalen Schüler/innenmerkmalen im Hinblick auf deren Gültigkeit für die beiden Gruppen geprüft.

Insgesamt bestätigen die Ergebnisse den kritischen Umstand, dass SmM überzufällig häufig Leistungsdefizite in Mathematik aufweisen. Analog zu den bisherigen Befunden (Faber et al., 2011; Shajek et al., 2006) erzielten SmM signifikant niedrigere Testleistungen als SoM. Dass daraus nicht zwangsläufig die Schlussfolgerung gezogen werden kann, dass bei SmM ebenfalls ungünstigere emotional-motivationale Lernvoraussetzungen vorliegen, konnte in dieser Untersuchung auch für die Gruppe von Schweizer Realschüler/innen (7. Klassenstufe) weitgehend bestätigt werden. Zwar indizieren die vorliegenden Befunde einen Zusammenhang zwischen niedrigeren Mathematikleistungen und höheren Angstwerten von SmM, allerdings weisen die Ergebnisse auch darauf hin, dass bei SmM vergleichsweise günstige emotional-motivationale Voraussetzungen in Mathematik bestehen (siehe auch Shajek et al., 2006). Dies bestätigt frühere Forschungsarbeiten, die im Hinblick auf die Lernfreude und das Fähigkeitskonzept ergeben haben, dass SmM zu Optimismus neigen (Faber et al., 2011). In der vorliegenden Untersuchung zeigt sich dieser Unterschied allerdings nur für die selbstbestimmte Motivation, wogegen sich die Mittelwerte im Fähigkeitsselbstkonzept und in der Lernfreude zwischen SmM und SoM nicht signifikant unterscheiden.

Die Diskrepanz zwischen SmM und SoM ist besonders stark bei Schüler/innen der ersten Generation. Wie auch bei den von Stanat und Christensen (2006) berichteten Befunden, weisen diese Schüler/innen zugleich die höchsten Werte im Fähigkeitsselbstkonzept und in der Angst auf. 
Die im Strukturgleichungsmodell dargestellten Befunde bestätigen weitgehend die in der KWT (Pekrun, 2006) postulierten fachspezifischen Zusammenhänge (Leistung $\rightarrow$ Fähigkeitsselbstkonzept $\rightarrow$ Emotion $\rightarrow$ Motivation im Fach Mathematik). Die vorliegende Untersuchung reiht sich demnach in Arbeiten zur Überprüfung der KWT in verschiedenen Lehr-Lern-Kontexten ein und testet sie gruppenbezogen für jugendliche Schüler/innen mit und ohne Migrationshintergrund. Die Ergebnisse deuten darauf hin, dass die Beziehungen zwischen Leistung, Fähigkeitsselbstkonzept, Emotion und Motivation gruppenunabhängig gelten, auch wenn deren mittlere Stärke zwischen den Gruppen (geringfügig) variiert. Die Befunde können als (erste) Bekräftigung der Gültigkeit der Merkmalsbeziehungen, wie sie in der KWT spezifiziert werden, interpretiert werden. Folgeuntersuchungen mit umfassendere Stichproben, in denen die Universalität der KWT über verschiedene Gruppen hinweg - ohne Power-Einschränkungen - getestet wird, sind jedoch notwendig.

Entgegen ursprünglicher Erwartungen lässt sich kein direkter Zusammenhang zwischen der Testleistung und dem positiv emotionalen Erleben und der selbstbestimmte Motivation nachweisen. Diesbezüglich wäre es interessant zu untersuchen, ob sich Schulnoten als Leistungsindikator als stärker prädiktiv erweisen würden. Marsh, Trautwein, Lüdtke, Köller und Baumert (2005) belegen für das Fähigkeitsselbstkonzept und das Interesse an Mathematik, dass der Einfluss der Noten deutlich grösser ist als jener von externen standardisierten Testleistungen. Die Autoren begründen dies damit, dass Schulnoten eine stärkere Rückmeldefunktion erfüllen und damit von den Schüler/innen direkt für soziale Leistungsvergleiche verwendet werden können. Diese Begründung ist auch für die vorliegende Studie naheliegend. Zudem ist zu erwähnen, dass die Schüler/innen kein Feedback auf den Leistungstest erhalten haben.

Weiter zeigt sich kein signifikanter Zusammenhang zwischen dem HISEI und der Testleistung in Mathematik. Dieser Befund irritiert zunächst, da die international vergleichende Bildungsforschung wiederholt belegt, dass die Schulleistung eng an die Herkunft der Schüler/innen geknüpft ist (z.B. Moser, 2005). Eine mögliche Erklärung, warum dieser Zusammenhang in der vorliegenden Studie nicht gefunden wurde, könnte in der relativen Homogenität der Stichprobe liegen. Die Varianz des HISEI ist durch die Fokussierung auf Realschüler/innen eingeschränkt, da Kinder aus bildungsnäheren Elternhäusern diesen Schultyp seltener besuchen. Interessant in diesem Zusammenhang wäre auch, die Schulnoten als Leistungsindikator im Zusammenhang mit dem HISEI $\mathrm{zu}$ untersuchen. $\mathrm{Zu}$ erwarten wäre etwa, dass Familien mit einem höheren sozioökonomischen Status auf schwache Schulleistungen ihrer Kinder proaktiv reagieren und zum Beispiel Nachhilfeunterricht in Anspruch nehmen. So ergibt eine Studie von Wolter und Hof (2012), dass Schüler/innen, deren Eltern eine akademische Bildung aufweisen, viermal so häufig Nachhilfe erhalten als Schüler/innen, deren Eltern über einen Berufsbildungsabschluss verfügen.

Hervorzuheben ist, dass die Ergebnisse der vorliegenden Studie fachspezifisch für das Fach Mathematik zu deuten sind. Aufgrund zahlreicher empirischer 
Befunde wird angenommen, dass etwa das verbale Fähigkeitsselbstkonzept von SmM in der Regel niedriger ist im Vergleich zu SoM (Faber et al., 2011; Shajek et al., 2006). Ähnliches ist für die Lernfreude oder die Motivation zu erwarten. Die fachspezifische Betrachtung von motivational-emotionalen Aspekten sowie der Leistung unter Berücksichtigung des Migrationshintergrundes scheint daher notwendig, um die Frage nach den Unterschieden zwischen SoM und SmM differenziert beantworten zu können.

Die Studie geht mit einigen Limitationen einher. Das Zusammenspiel der angeführten Schüler/innenmerkmale wurde in der vorliegenden Untersuchung unidirektional - basierend auf einem querschnittlichen Datensatz - modelliert. Künftig sollte in Längsschnittstudien den erwarteten Wechselwirkungen näher nachgegangen werden. Als Einschränkung muss des Weiteren eingeräumt werden, dass aufgrund der geringen Stichprobengrösse a) die Ergebnisse (insbesondere der Vergleich zwischen der ersten und zweiten Generation) und die damit einhergehende geringe Power ${ }^{3}$ mit Zurückhaltung zu interpretieren sind, b) keine Überprüfung von Messinvarianz durchgeführt werden konnte und c) nicht zwischen unterschiedlichen Herkunftsländern in der Gruppe der SmM unterschieden werden konnte. Da davon ausgegangen werden muss, dass SmM keine homogene Gruppe darstellen (z.B. bezogen auf die Bildungsaspirationen), sollten in zukünftigen Forschungsarbeiten die Herkunftsländer von SmM stärker Berücksichtigung finden.

Zusammenfassend lässt sich festhalten, dass die vorliegende Studie die Befundlage zu den Unterschieden von SmM und SoM in der Schweiz erweitert, da sie relevante schulbezogene Schüler/innenmerkmale im Fach Mathematik auf Realniveau im Jugendalter (einer besonders kritischen Phase bezogen auf die Entwicklung der schulischen Lernmotivation und einer positiven Haltung gegenüber der Schule) untersucht. Sie bestätigt den Befund, dass SmM zwar geringere Leistungen in Mathematik erzielen, jedoch in einigen emotional-motivationalen Merkmalen günstigere Voraussetzungen mitbringen, insbesondere in der selbstbestimmten Motivation. In diesem Zusammenhang stellt sich die Frage, wie sich die Beziehung zwischen dem motivationalen Potential - welches bei SmM in einem höheren Ausmass vorhanden ist - und der Leistung stärken lässt. Mit Blick auf die gezielte Förderung von SmM könnte diese positive Lernvoraussetzung als Ressource in der konkreten Arbeit in Schule und Unterricht genutzt werden, indem z.B. durch zusätzliche Angebote wie Lerngruppen in Mathematik die Leistungsentwicklung spezifisch unterstützt wird.

\section{Anmerkungen}

1 im Ausland geborene Schüler/innen mit im Ausland geborenen Eltern (Bauer, Ramseier \& Blum, 2014).

2 Die interkantonale Vereinbarung über die Harmonisierung der obligatorischen Schule in der Schweiz ist ein interkantonales Konkordat mit dem Ziel der Vereinheitlichung der obligatorischen Schule (Kindergarten, Primarschule und Sekundarstufe 1) (Bauer et al., 2014). 
3 Um die nicht signifikanten Ergebnisse zwischen der ersten und zweiten Generation der durchgeführten Analysen angesichts der gegebenen Stichprobengrösse beurteilen zu können, wurden Power Analysen mittels der Software G*Power berechnet (Mayr, Erdfelder, Buchner \& Faul, 2007). Bezogen auf die motivational-emotionalen Merkmale ergibt die Poweranalyse für ein $\alpha$-Fehlerniveau von .05 für eine Power von $1-\beta=.95$ und eine erwartete Effektstärke von $\mathrm{p}=.50$ eine Power von 72.55 ; für eine erwartete Effektstärke von $\mathrm{p}=.20$ eine Power von 17.60. Die Wahrscheinlichkeit, bei einem $t$-Test einen tatsächlichen kleinen Mittelwertsunterschied zwischen Schüler/innen der ersten und der zweiten Generation zu entdecken, ist demnach gering, weshalb der Effektstärke besondere Bedeutung zukommt. Zudem ist zu erwähnen, dass die berechnete Power von unabhängigen Messungen ausgeht. Im vorliegenden Fall wurden jedoch Schüler/innen in Klassen befragt.

\section{Literatur}

Allraum, J. (2015). Bildungsstatistik Kanton Bern: Basisdaten 2014. Bern: Abteilung Bildungsplanung und Evaluation (BiEv) Erziehungsdirektion des Kantons Bern.

Bauer, C., Ramseier, E. \& Blum, D. (2014). PISA 2012: Porträt des Kantons Bern (deutschsprachiger Teil). Bern: Forschungsgemeinschaft PISA Deutschschweiz.

Billmann-Mahecha, E. \& Tiedemann, J. (2006). Übergangsempfehlung als kritisches Lebensereignis: Migration, Übergangsempfehlung und Fähigkeitsselbstkonzept. In A. Schründer-Lenzen (Hrsg.), Risikofaktoren kindlicher Entwicklung. Migration, Leistungsangst und Schulübergang (S. 193-207). Wiesbaden, Deutschland: Verlag für Sozialwissenschaften.

Breit, S. (2009). Kompetenzen von Jugendlichen mit Migrationshintergrund. In C. Schreiner \& U. Schwantner (Hrsg.), PISA 2006. Österreichischer Expertenbericht zum Naturwissenschaftsschwerpunkt (S. 146-158). Graz, Österreich: Leykam.

Buholzer, A., Ottiger, A. \& von Büren, A. (2008). "Weiterentwicklung der Sekundarstufe I". Bericht zur Evaluation der Sekundarstufe I im Kanton Luzern. Band I (Forschungsbericht Nr. 12). Luzern: der Pädagogischen Hochschule Zentralschweiz, Hochschule Luzern.

Deci, E. L. \& Ryan, R. M. (1993). Die Selbstbestimmungstheorie der Motivation und ihre Bedeutung für die Pädagogik. Zeitschrift für Pädagogik, 39(2), 223-238.

Faber, G., Tiedemann, J. \& Billmann-Mahecha, E. (2011). Selbstkonzept und Lernfreude in der Grundschulmathematik: Die Bedeutung von Migration und Geschlecht. Längsschnittliche Ergebnisse aus der Hannoverschen Grundschulstudie. Heilpädagogische Forschung, 37(3), 127-143.

Fend, H. (1997). Der Umgang mit Schule in der Adoleszenz. Aufbau und Verlust von Lernmotivation, Selbstachtung und Empathie. Bern: Huber.

Ganzeboom, H. B., De Graaf, P. M. \& Treiman, D. J. (1992). A standard international socio-economic index of occupational status. Social science research, 21(1), 1-56.

Geiser, C. (2010). Datenanalyse mit Mplus. Eine anwendungsorientierte Einführung. Wiesbaden, Deutschland: VS.

Götz, T., Pekrun, R., Zirngibl, A., Jullien, S., Kleine, M., vom Hofe, R. \& Blum, W. (2004). Leistung und emotionales Erleben im Fach Mathematik: Längsschnittliche Mehrebenenanalysen. Zeitschrift für Pädagogische Psychologie, 18(3/4), 201-212.

Hascher, T. \& Hagenauer, G. (2011). Schulisches Wohlbefinden im Jugendalter-Verläufe und Einflussfaktoren. In A. Ittel, H. Merkens \& L. Stecher (Hrsg.), Jahrbuch Jugendforschung (S. 15-45). Wiesbaden, Deutschland: Verlag für Sozialwissenschaften.

Helmke, A., Hosenfeld, I., Schrader, F. \& Wagner, W. (2002). Sozialer und sprachlicher Hintergrund. In A. Helmke \& R. S. Jäger (Hrsg.), Das Projekt MARKUS. Mathematik-Gesamterhebung Rheinland-Pfalz: Kompetenzen, Unterrichtsmerkmale, Schulkontext (S. 71-153). Landau, Deutschland: Verlag Empirische Pädagogik. 
Hof, S. \& Wolter, C. (2012). Bezahlte Nachhilfe: Gekaufte Bildung als Lerndoping? Bildung Schweiz (9), 34-35.

Hosenfeld, I. (2002). Kausalitätsüberzeugungen und Schulleistungen. Münster: Waxmann.

Jerusalem, M. (1988). Selbstwert, Ängstlichkeit und Sozialklima von jugendlichen Migranten. Zeitschrift für Sozialpsychologie, 19, 53-62.

Lehmann, R., Gänsfuss, R. \& Peek, R. (2011). LAU 7. Aspekte der Lernausgangslage und der Lernentwicklung-Klassenstufe 7. In Behörde für Schule und Berufsausbildung (Hrsg.), $L A U$ - Aspekte der Lernausgangslage und der Lernentwicklung. Klassenstufen 5, 7 und 9 (S. 121-285). Münster, Deutschland: Waxmann.

Marsh, H. W., Trautwein, U., Lüdtke, O., Köller, O. \& Baumert, J. (2005). Academic selfconcept, interest, grades, and standardized test scores: reciprocal effects models of causal ordering. Child development, 76(2), 397-416.

Mayr, S., Erdfelder, E., Buchner, A. \& Faul, F. (2007). A short tutorial of GPower. Tutorials in Quantitative Methods for Psychology, 3, 51-59.

Mega, C., Ronconi, L. \& De Beni, R. (2014). What makes a good student? How emotions, self-regulated learning, and motivation contribute to academic achievement. Journal of Educational Psychology, 106(1), 121-131.

Meyer, T. (2003). Jugendliche mit Migrationshintergrund. Wege in die nachobligatorische Ausbildung. Die ersten zwei Jahre nach Austritt aus der obligatorischen Schule. Zwischenergebnisse des Jugendlängsschnitts TREE. EDK/CDIP, BFS/OFS \& Neuchâtel, BFS/OFS.

Moser, U. (2005). Kontextmerkmale des Bildungssystems und ihre Bedeutung für die Mathematikleistungen. In Forschungsgemeinschaft PISA Deutschschweiz/FL (Hrsg,), PISA 2003: Analysen und Porträts für Deutschschweizer Kantone und das Fürstentum Liechtenstein. Zusammenfassung der wichtigsten Ergebnisse (S. 105-140). Zürich: KDMZ.

Müller, F. H., Hanfstingl, B. \& Andreitz, I. (2007). Skalen zur motivationalen Regulation beim Lernen von Schülerinnen und Schülern. Adaptierte und ergänzte Version des Academic Self-Regulation Questionnaire (SRQ-A) nach Ryan \& Connell. Wissenschaftliche Beiträge aus dem Institut für Unterrichts- und Schulentwicklung. Klagenfurt: Alpen-Adria-Universität.

Muthén, L. \& Muthén, B. (1998-2012). Mplus userss guide (7th ed.). Los Angeles, CA: Muthén \& Muthén.

Pekrun, R. (2006). The control-value theory of achievement emotions: Assumptions, corollaries, and implications for educational research and practice. Educational Psychology Review, 18(4), 315-341.

Pekrun, R., Götz, T., Titz, W. \& Perry, R. P. (2002). Academic emotions in students's self-regulated learning and achievement: A program of quantitative and qualitative research. Educational Psychologist, 37, 91-106.

Pekrun, R. \& Götz, T (2006). Emotionsregulation: Vom Umgang mit Prüfungsangst. In H. Mandl \& H.F. Friedrich (Hrsg.), Handbuch Lernstrategien, 248-258. Göttingen, Deutschland: Hogrefe.

Pekrun, R., Götz, T. \& Frenzel, A. (2005). Achievement Emotions Questionnaire-Mathematics (AEQ-M) user's manual. University of Munich: Department of Psychology.

Rakoczy, K., Klieme, E. \& Pauli, C. (2008). Die Bedeutung der wahrgenommenen Unterstützung motivationsrelevanter Bedürfnisse und des Alltagsbezugs im Mathematikunterricht für die selbstbestimmte Motivation. Zeitschrift für Pädagogische Psychologie, 22(1), 25-35.

Rheinberg, F. \& Wendland, M. (2002). Veränderung der Lernmotivation in Mathematik: Eine Komponentenanalyse auf der Sekundarstufe I. In M. Prenzel \& J. Doll (Hrsg.), Bildungsqualität von Schule: Schulische und ausserschulische Bedingungen mathematischer, naturwissenschaftlicher und überfachlicher Kompetenzen (S. 308-319). Weinheim, Deutschland: Beltz. 
Roebers, C. M., Mecheril, A. \& Schneider, W. (1998). Migrantenkinder in deutschen Schulen. Eine Studie zur Persönlichkeitsentwicklung. Zeitschrift für Pädagogik, 44(5), 723-736.

Roebers, C. M. \& Schneider, W. (1999). Self-concept and anxiety in immigrant children. International Journal of Behavioral Development, 23(1), 125-147.

Schiepe-Tiska, A. \& Schmidtner, S. (2013). Mathematikbezogene emotionale und motivationale Orientierungen, Einstellungen und Verhaltensweisen von Jugendlichen in PISA 2012. In M. Prenzel, C. Sälzer, E. Klieme \& O. Köller (Hrsg.), PISA 2012: Fortschritte und Herausforderungen in Deutschland (S. 100-121). Münster, Deutschland: Waxmann.

Schöber, C., Retelsdorf, J. \& Köller, O. (2015). Verbales schulisches Selbstkonzept und sprachliche Leistungen in Gruppen mit und ohne Migrationshintergrund. Psychologie in Erziehung und Unterricht, 62(2), 89-105.

Schrader, F. \& Helmke, A. (2002). Motivation, Lernen und Leistung. In A. Helmke \& R. S. Jäger (Hrsg.), Das Projekt MARKUS. Mathematik-Gesamterhebung Rheinland-Pfalz: Kompetenzen, Unterrichtsmerkmale, Schulkontext (S. 257-323). Landau, Deutschland: Verlag Empirische Pädagogik.

Schwantner, U., Toferer, B. \& Schreiner, C. (2013). PISA 2012. Internationaler Vergleich von Schülerleistungen. Erste Ergebnisse. Mathematik. Lesen. Naturwissenschaft. Graz, Österreich: Leykam.

Schwarzer, R., Lange, B. \& Jerusalem, M. (1981). Selbstkonzept und Ängstlichkeit bei deutschen und ausländischen Grundschülern. Unterrichtswissenschaft, 9(2), 112-119.

Shajek, A., Lüdtke, O. \& Stanat, P. (2006). Akademische Selbstkonzepte bei Jugendlichen mit Migrationshintergrund. Unterrichtswissenschaft, 34(2), 125-145.

Shuman, V. \& Scherer, K. R. (2014). Concepts and structures of emotions. In R. Pekrun \& L. Linnenbrink-Garcia (Hrsg.), International handbook of emotions in education (S. 13-35). New York, NY: Taylor \& Francis

Stanat, P. \& Christensen, G. (2006). Schulerfolg von Jugendlichen mit Migrationshintergrund im internationalen Vergleich: Eine Analyse von Voraussetzungen und Erträgen schulischen Lernens im Rahmen von PISA 2003. Berlin: Bundesministerium für Bildung und Forschung (BMBF).

Stanat, P., Rauch, D. \& Segeritz, M. (2010). Schülerinnen und Schüler mit Migrationshintergrund. In E. Klieme, C. Artelt, J. Hartig, N. Jude, O. Köller, M. Prenzel, W. Schneider \& P. Stanat (Hrsg.), PISA 2009. Bilanz nach einem Jahrzehnt (S. 200-230). Münster, Deutschland: Waxmann.

Stanat, P., Segeritz, M \& Christensen, G. (2010). Schulbezogene Motivation und Aspiration von Schülerinnen und Schülern mit Migrationshintergrund. In W. Bos, E. Klieme \& O. Köller (Hrsg.), Schulische Lerngelegenheiten und Kompetenzentwicklung. Festschrift für Jürgen Baumert (S 31-57). Münster, Deutschland: Waxmann.

Wälti, B. (2014). Alternative Leistungsbewertung in der Mathematik: mathematische Beurteilungsumgebungen: theoretische Auseinandersetzung und empirische Studie. Bern: Schulverlag plus.

Schlagworte: Selbstbestimmungstheorie, Kontroll-Wert-Theorie, Migrationshintergrund, Motivation, Emotionen, Selbstkonzept, Mathematik 


\section{Interaction entre le rendement en mathématiques, la motivation des élèves et l'apprentissage émotionnel au degré secondaire 1 - une comparaison entre élèves issus ou non de familles immigrées en Suisse.}

\section{Résumé}

Sur la base du constat que les élèves issus de familles immigrées en Suisse obtiennent des résultats plus faibles en mathématiques que leurs camarades sans antécédent migratoire, la présente étude examine si des différences analogues existent pour la motivation et l'apprentissage émotionnel, deux facteurs déterminants pour la performance des élèves. En plus, et fondé sur la "control-value theory», est étudiée la relation entre ces facteurs et les résultats obtenus en mathématiques. L'étude ( $\mathrm{N}=415$ élèves section générale, $7 \mathrm{e}$ classe) confirme le rendement inférieur des élèves avec antécédent migratoire, accompagné d'une motivation autodéterminée supérieure et un sentiment d'angoisse augmenté. Un bon rendement est associé avec le concept de soi, qui à son tour prédit une expérience émotionnelle positive (joie haute et basse anxieté) et une motivation autodéterminée.

Mots clés: Théorie de l'autodétermination, control-value theory, antécédent migratoire, motivation, émotions, concept de soi, mathématiques

\section{Interazione tra il rendimento in matematica, la motivazione degli studenti e l'apprendimento emozionale al livello secondario 1 - un paragone tra gli studenti con o senza contesto migratorio in Svizzera.}

\section{Riassunto}

Basato sulla relazione che gli studenti appartenenti a famiglie migranti ottengono risultati più deboli in matematica paragonati a quelli dei loro compagni senza contesto migratorio, il presente studio esamina se queste differenze si manifestino anche nella motivazione e nell'apprendimento emozionale, due fattori determinanti per il rendimento degli studenti. In oltre, sulla base della " controlvalue theory " si analizza il rapporto tra questi fattori e i risultati ottenuti in matematica. Lo studio ( $\mathrm{N}=415$ studenti della media generale, 7 a classe) conferma il rendimento inferiore degli studenti con contesto migratorio, associato a una motivazione autodeterminata superiore e un sentimenti d'angoscia aumentato. Un rendimento alto e correlato con l'autoconcetto in matematica il quale a sua volta prevede emozioni positive (grande gioia, bassa ansia) e una motivazione autodeterminata.

Parole chiave: Teoria dell'autodeterminazione; control-value theory, contesto migratorio, motivazione, emozioni, autoconcetto, matematica 


\section{The interplay between maths achievement, student motivation and learning emotions in secondary school - a comparison of students with and without an immigration background in Switzerland}

\section{Summary}

Based on the finding that students with an immigrant background in Switzerland reach considerably lower levels of math achievement than their peers without an immigrant background, the following study examines whether these differences are reflected in their emotional and motivational characteristics. In addition, on the basis of the control-value theory, we explore the relationship between these constructs and their link to math achievement. The results $(\mathrm{N}=415$ seventh grade basic level students) confirm the lower test score achievement of students with an immigration background, with simultaneous higher self-determined motivation and higher anxiety. High achievement is related to a high academic self-concept in math, which again explains a positive emotional pattern (high joy, low anxiety) and self-determined motivation.

Keywords: Self-determination-Theory, Control-Value-Theory, Immigration background, motivation, emotions, self-concept, mathematics 